\title{
¿'Pee' o ‘Bee'? Identificación por oyentes argentinos y norteamericanos de oclusivas iniciales del inglés con diferentes duraciones manipuladas de Voice Onset Time
}

\author{
'Pee' ou 'Bee'? Identificação, por ouvintes argentinos e norteamericanos, de plosivas iniciais do inglês com \\ diferentes durações manipuladas de Voice Onset Time
}

\section{Ubiratã Kickhöfel Alves}

Universidade Federal do Rio Grande do Sul - UFRGS - Porto Alegre - Rio Grande do Sul - Brasil

$$
\text { Pedro Luís Luchini }
$$

Universidad Nacional de Mar del Plata - UNMdP - Buenos Aires - Argentina

\section{Camila Motta-Avila}

Universidade Federal do Rio Grande do Sul - UFRGS - Porto Alegre - Rio Grande do Sul - Brasil

\begin{abstract}
Resumen: En este trabajo, investigamos la identificación de sonoridad de las oclusivas iniciales del inglés por parte de 24 oyentes argentinos (L1: Español Rioplatense) y 23 participantes norteamericanos. Sustentados en la premisa de que la percepción de los sonidos de una nueva lengua resulta en la atribución de diferentes valores asignados a las pistas acústicas que se muestran prioritarias en la lengua en cuestión (HOLT; LOTTO, 2006; IDEMARU; HOLT; SELTMAN, 2012; LEHET; HOLT, 2017), estudios previos (ALVES; LUCHINI, 2016, 2017a) demostraron que los oyentes argentinos, al contrario de los norteamericanos, no consideran el Voice Onset Time (VOT) como pista acústica principal para las distinciones de sonoridad de las oclusivas iniciales. Con base en estos estudios, en este trabajo los participantes fueron expuestos a diferentes duraciones manipuladas de VOT. A partir de oclusivas sordas iniciales aspiradas del inglés (con VOT largo), manipulamos la duración para contar no sólo con el VOT total, sino también con el $75 \%$, el $50 \%$ y el $25 \%$ de dicha duración, además del patrón VOT Cero. Los resultados confirman que el VOT constituye la pista prioritaria para la distinción de sonoridad entre los oyentes norteamericanos, revelando diferencias significativas entre los dos grupos en la mayoría de las condiciones de VOT testeadas. Los resultados también revelan que, a pesar de que el VOT no es la pista mayoritariamente seguida por los estudiantes argentinos, tal pista es, también, tomada en consideración en las decisiones de sonoridad por tales estudiantes, de forma combinada con otras pistas acústicas.
\end{abstract}

Palabras clave: percepción de L2, oclusivas iniciales, español rioplatense (L1), inglés (L2), voice onset time.

Resumo: Neste trabalho, investigamos a identificação dos padrões de sonoridade das plosivas iniciais do inglês por parte de 24 ouvintes argentinos (L1: Espanhol Rioplatense) e 23 participantes norte-americanos. Com base na premissa de que a percepção dos sons de uma nova língua implica novas atribuições de valor às pistas acústicas que desempenham um papel prioritário na língua em questão (HOLT; LOTTO, 2006; IDEMARU; HOLT; SELTMAN, 2012; LEHET; HOLT, 2017), estudos prévios (ALVES; LUCHINI, 2016, 2017a) demonstraram que os ouvintes argentinos, ao contrário dos norte-americanos, não consideram o Voice Onset Time (VOT) como pista acústica principal para as distinções de sonoridade das plosivas iniciais. Com base nesses estudos, neste trabalho os participantes foram expostos a diferentes durações manipuladas de VOT. A partir de plosivas surdas iniciais aspiradas do inglês (com VOT longo), manipulamos a duração de VOT de tais estímulos para contar não somente com a duração total, mas também com 75\%, 50\% e 25\% desta duração, além do padrão VOT Zero. Os resultados confirmam que o VOT constitui a pista prioritária para a distinção de sonoridade entre os ouvintes norte-americanos, revelando diferenças significativas entre os dois grupos na maioria das condições de VOT testadas. Os resultados também revelam que, apesar de o VOT não ser a pista majoritária entre os estudantes argentinos, tal pista é, também, levada em consideração nas distinções de sonoridade realizadas por tais estudantes, de modo a ser combinada com outras pistas acústicas.

Palavras-chave: percepção de L2, plosivas iniciais, espanhol rio-platense (L1), inglês (L2), voice onset time. 


\section{Introducción}

El trabajo que aquí se presenta es el resultado de una trayectoria de cinco años de cooperación académica, en el ámbito del área de Adquisición Fonético-Fonológica de Lenguas Extranjeras, entre la Universidad Federal de Rio Grande do Sul (UFRGSBrasil) y la Universidad Nacional de Mar del Plata (UNMdP-Argentina). A través del interés del primer y del segundo autor de este trabajo acerca del proceso de adquisición de la fonología del inglés, tanto por estudiantes argentinos como por brasileños, la referida cooperación, que ya ha resultado en dos instancias postdoctorales y orientaciones compartidas de tesis de doctorado, hizo posible que delineáramos las principales dificultades fonético-fonológicas de tales estudiantes en el proceso de construcción de la fonología de la lengua inglesa. De esta forma, de la integración de los grupos de investigación 'Labico Laboratorio de Bilingüismo y Cognición' (UFRGS), y 'Cuestiones del Lenguaje' (UNMdP), resultó una serie de trabajos publicados en capítulos de libros y revistas nacionales e internacionales (LUCHINI; GARCÍA-JURADO; ALVES, 2015; ALVES; LUCHINI, 2016; 2017a; 2017b). Tal diálogo académico nos trajo a la presente etapa de investigación, cuyos resultados se expresan en el presente estudio.

Entre los diversos fenómenos fonéticofonológicos cuya adquisición en lengua inglesa ha sido investigada de forma conjunta por los dos grupos de investigación antes mencionados, los procesos de producción de las oclusivas iniciales sordas del inglés, así como la percepción de diferentes patrones de Voice Onset Time (en adelante, VOT) por tales estudiantes, ha recobrado particular importancia en una serie de trabajos (ALVES; MOTTA, 2014; MOTTA, 2014; ALVES; ZIMMER, 2015; SCHWARTZHAUPT; ALVES; FONTES, 2015; ALVES; LUCHINI, 2016, 2017a, 2017b; MOTTAAVILA, 2017). Considerando que los sonidos del habla se caracterizan por una gran cantidad de pistas acústicas que actúan en conjunto para la identificación de un determinado segmento en una lengua determinada, y que cada lengua atribuye distintos valores al grado de funcionalidad de una pista en relación a otra ('cue weighting', cf. HOLT; LOTTO, 2006; IDEMARU; HOLT; SELTMAN, 2012; LEHET; HOLT, 2017), los trabajos mencionados anteriormente han enfocado en el valor del patrón VOT Positivo (que corresponde a la sensación auditiva de 'aspiración') para la identificación del estatus de sordo-sonoro de las oclusivas iniciales en inglés, en portugués brasileño y en la variedad de español rioplatense. En inglés, esta pista asume un carácter fundamental para las distinciones de sonoridad entre pares mínimos tales como 'pill vs. 'bill, 'tip' vs. 'dip' o 'Kate' vs. 'gate', de modo que la presencia de un VOT Positivo es el factor identificador del segmento oclusivo como sordo. Por su parte, hablantes argentinos y brasileños, en sus idiomas maternos, producen la oclusiva inicial sonora con prevoceo y la sorda con un patrón de VOT Cero (es decir, sin aspiración), de modo que la producción de la aspiración puede ser considerada un desafío para estos estudiantes.

Estudios realizados con oyentes norteamericanos de inglés (cf. MOTTA, 2014; SCHWARTZHAUPT, 2015; SCHWARTZHAUPT; ALVES; FONTES, 2015) confirman la previsión de la literatura (LISKER; ABRAMSON, 1964; JENKINS, 2000; CELCE-MURCIA et al., 2010; ABRAMSOM; WHALEN, 2017) respecto a la importancia de esta pista, una vez que producciones manipuladas de oclusivas iniciales sin aspiración revelaron que dicha ausencia resultó en la identificación de tal segmento como sonoro. Verificamos, por lo tanto, la necesidad de que los estudiantes extranjeros de lengua inglesa aprendan a realizar la debida aspiración para el establecimiento de las distinciones de sonoridad.

Sin embargo, diversos estudios llevados a cabo en Brasil y en Argentina, tanto con participantes brasileños (ZIMMER, 2004; REIS; NOBREOLIVEIRA, 2008; FRANÇA, 2015; SCHWARTZHAUPT, 2013; PRESTES, 2013; ALVES; ZIMMER, 2015) como con argentinos (ALVES; LUCHINI, 2017a, 2017b; ALVES; SCHERESCHEWSKY; LUCHINI, en prensa), 
muestran que los estudiantes de ambas nacionalidades presentan dificultades en la producción de la aspiración, lo que da lugar a problemas en la inteligibilidad de tales estímulos por parte de los oyentes norteamericanos (MOTTA, 2014; SCHWARTZHAUPT, 2015; SCHWARTZHAUPT; ALVES; FONTES, 2015; ALVES; LUCHINI, 2016; MOTTA-AVILA, 2017). Por lo tanto, nos pareció relevante discutir si la ausencia de aspiración era el resultado de las dificultades perceptivas por parte de los estudiantes, para verificar si eran capaces de atribuir a la presencia/ausencia de aspiración el estatus de pista acústica fundamental para la identificación de los sonidos sordos y sonoros.

En Alves; Motta (2014) y Alves; Zimmer (2015), verificamos que los estímulos sordos no manipulados, con la oclusiva con aspiración, eran fácilmente identificados como sordos por los brasileños. Además, en estos estudios, contamos, también, con la exposición de los estudiantes brasileños a otros tres distintos patrones de VOT: (i) el patrón VOT Negativo (con pre-voceo); (ii) el patrón VOT Cero, referente a la producción de las oclusivas /b/, /d/, /g/ por hablantes nativos ${ }^{1}$; (iii) un patrón manipulado, denominado de Cero Artificial, que resultó de la eliminación de la aspiración de las oclusivas /p/, /t/ y /k/. A partir de la manipulación de la aspiración, contamos con un patrón híbrido: al mismo tiempo en que la pista referente al VOT presentaba un patrón (VOT Cero) característico de una oclusiva sonora, todas las demás pistas acústicas que configuran tal segmento (tal como la fuerza de explosión del segmento y el FO de la vocal siguiente) conservaban las características proprias de un segmento sonoro.

De los tres patrones anteriormente citados, como se esperaba en función de la propia L1 de los participantes, el patrón VOT Negativo fue categóricamente identificado como sonoro. Por su

\footnotetext{
1 Es importante mencionar que, en la lengua inglesa, las oclusivas sonoras iniciales /b/, /d/ y /g/ son producidas, variablemente, tanto con el patrón VOT Negativo como con el patrón VOT Cero (LISKER; ABRAHAMSON, 1964). Este último patrón puede representar dificultades perceptivas para los estudiantes brasileños y argentinos, porque caracteriza las oclusivas sordas iniciales de las dos lenguas en cuestión (como en las palabras 'pato', 'toda' y 'cama', cognadas en los dos idiomas latinos).
}

parte, encontramos resultados interesantes para los patrones VOT Cero Natural y VOT Cero Artificial, ya que ambos patrones, con valores similares de VOT, fueron identificados de forma diferente: mientras que el patrón natural ya tendía a ser preferentemente identificado como sonoro, el patrón artificial, con todas las pistas características de las oclusivas sordas con excepción de la propia aspiración, tendía a ser predominantemente identificado como sordo.

Los resultados anteriores constituyen evidencia de que los estudiantes brasileños estaban utilizando otras pistas acústicas, además del VOT, para distinguir las oclusivas sordas de las sonoras del inglés. En otras palabras, considerándose la concepción de cue weighting (HOLT; LOTTO, 2006; IDEMARU; HOLT; SELTMAN, 2012; LEHET; HOLT, 2017), el VOT no resultó ser la pista prioritaria para las distinciones de sonoridad. El resultado de este trabajo se opone al verificado en Schwartzhaupt; Alves; Fontes (2015), en el que utilizamos el mismo instrumento perceptual con oyentes nativos de inglés, residentes de Pensilvania, Estados Unidos: en dicho estudio, tanto el patrón VOT Cero Natural como el VOT Cero Artificial fueron identificados como sonoros, confirmando el estatus prioritario de esta pista en la lengua-meta.

En vista de estos resultados nos preguntamos si, con estudiantes argentinos de inglés (cuya L1 también cuenta con los mismos patrones de VOT del portugués de Brasil), los resultados serían similares. De esta forma, en Alves; Luchini (2016) usamos el mismo instrumento que empleamos en los trabajos anteriores, pero con estudiantes de nivel C1 de dominio de inglés. Los resultados fueron semejantes a los obtenidos con los estudiantes brasileños, porque se observaron diferentes índices de respuesta para los estímulos que exhibían el patrón VOT Cero Natural y el VOT Cero Artificial. Estos datos demuestran que, en términos pedagógicos, tanto estudiantes argentinos como brasileños deben prestar mayor atención a la importancia de la pista de VOT. Tal aseveración nos motivó a explorar los efectos de la instrucción explícita y del entrenamiento perceptivo 
entre estudiantes de ambas nacionalidades (ALVES; LUCHINI; 2017a; ALVES; KAMPFF, en prensa).

Aunque los estudios antes citados han evidenciado claramente que el VOT no corresponde a la pista principal entre estudiantes brasileños y argentinos de inglés, cabe formular una salvedad a los patrones de VOT probados en estos experimentos. En el instrumento arriba mencionado, sólo se verificó el papel de la presencia del intervalo total (VOT Positivo) o de la ausencia prácticamente ${ }^{2}$ total (VOT Cero Natural o Cero Artificial) de la aspiración, lo que demuestra que, en los casos de estímulo con VOT Cero, otras pistas asumieron un estatus primordial para las distinciones de sonoridad entre los estudiantes. Sin embargo, nos preguntábamos si con diferentes duraciones de VOT, que fueran más allá de la simple 'ausencia' o 'presencia' plena de aspiración, la pista de VOT podría ejercer algún efecto proprio por sí sola o en conjunto con las demás pistas acústicas en la identificación perceptiva de los estudiantes.

En línea con este interrogante, en Motta-Avila (2017) se investigaron los efectos de múltiples manipulaciones de duración de VOT en la identificación de las oclusivas del inglés por oyentes norteamericanos y estudiantes brasileños. Para tal verificación, a partir de estímulos con oclusivas que exhibían VOT Positivo (aspirado), fueron retiradas porciones de VOT referentes al 25\% de la duración total, de modo que contáramos con diferentes patrones de VOT, correspondientes a la duración de la aspiración (100\%, 75\%, 50\%, 25\% y 0\%, considerándose el VOT total). Los resultados revelaron que los participantes estadounidenses son indiscutiblemente más sensibles al corte de la pista acústica de VOT que los participantes brasileños, independientemente del nivel de competencia en lengua inglesa de tales estudiantes (básico, intermedio y avanzado). Se demuestra, nuevamente, que los hablantes nativos de inglés, a diferencia de los oyentes brasileños, toman el VOT como pista

\footnotetext{
2 El término 'prácticamente' se debe al hecho de que el patrón Cero Artificial no necesariamente corresponde a un intervalo de VOT de duración de 0 ms, sino un patrón con una duración muy próxima a cero.
}

acústica prioritaria. Los oyentes brasileños, una vez más, tienden a identificar los patrones manipulados como sordos.

En el presente artículo, nos indagamos si la aplicación del test de identificación empleado en Motta-Avila (2017) a estudiantes argentinos brindará resultados diferentes a los previamente encontrados con estudiantes brasileños. En caso afirmativo, llegaremos a la conclusión de que, aunque el VOT no corresponda a la pista prioritaria identificada por los oyentes brasileños y argentinos, tal pista puede ejercer, entre los estudiantes del grupo hispánico, un papel más relevante en la identificación de sonoridad de los segmentos oclusivos iniciales, aunque en combinación con otras pistas acústicas. Por su parte, en el caso de encontrar resultados similares a los de los brasileños (es decir, si verificamos que, independientemente de la manipulación, estos estudiantes seguirán identificando tales segmentos manipulados como sordos), tendremos evidencias adicionales de que los estudiantes de ambos sistemas de L1 no toman el VOT como una pista relevante para la distinción de sonoridad. Participaron en este estudio 24 estudiantes argentinos de inglés, con un nivel de competencia lingüística equivalente al C1, según lo indica la escala del Marco Común Europeo de Referencia Lingüística ${ }^{3}$. Los participantes realizaron el mismo experimento de identificación de consonantes con múltiples manipulaciones de VOT empleado en Motta-Avila (2017). Como control, se tomaron los datos de los 23 participantes norteamericanos del estudio previo. En esta investigación pretendemos verificar si (i) habrá diferencias en las respuestas de los participantes en función del patrón de manipulación del estímulo (VOT 0, 25\%, 50\%, $75 \%$ y $100 \%$ del intervalo total de VOT); (ii) habrá diferencias en los índices de identificación en función del sistema de L1 del oyente (hablantes nativos y no nativos).

\footnotetext{
${ }^{3}$ A diferencia del estudio de Motta-Ávila (2017), que contó con la participación de brasileños con tres diferentes niveles de competencia lingüística de inglés, en el presente trabajo contamos con la participación de solo un grupo de estudiantes, con nivel avanzado. Esta decisión se debió a que los datos de los estudios de esta autora no evidenciaron diferencias en función de los diferentes niveles de competencia lingüística de sus participantes.
} 
Por medio de esta investigación, esperamos contribuir a la concepción de la propuesta de ponderación de pistas acústicas (cue weighting), y destacar argumentos empíricos adicionales que sirvan como punto de partida para discutir la importancia y el estatus de la pista de VOT entre estudiantes argentinos de inglés.

\section{Método}

\subsection{Participantes}

Participaron 24 estudiantes argentinos, residentes en la ciudad de Mar del Plata-Argentina, siendo, por lo tanto, hablantes de la variedad de español rioplatense (L1), matriculados regularmente en clases de inglés de nivel avanzado de dos instituciones de enseñanza superior de la ciudad de Mar del Plata. Siguiendo la misma metodología empleada en Alves; Luchini (2017a), de acuerdo con el nivel del curso en que se encontraban tales participantes, y de sus propios relatos de autoevaluación, todos los participantes presentaban mínimamente un nivel de competencia lingüística equivalente al $\mathrm{C} 1$ según el Marco Común Europeo de Referencia de Lenguas. Al momento de la recolección de datos, los participantes tenían entre 19 y 24 años de edad. En sus relatos, informaron que todos habían completado estudios de inglés en institutos 0 academias de enseñanza de lenguas extranjeras, previos al inicio de sus carreras universitarias. Agregaron, además, que el inglés era la única lengua adicional que manejaban. A su vez, los datos de los 23 participantes norteamericanos investigados em Motta-Avila (2017) fueron empleados como grupo control. Estos participantes también se encontraban cursando sus carreras de grado o tomando cursos de posgrado en la universidad. Con respecto a su formación en lenguas extranjeras, reportaron no contar con alto dominio de una lengua adicional, por lo que se los consideró como monolingües (hablantes de inglés, únicamente).

Los participantes de ambos grupos fueron contactados de diversas maneras: personalmente, virtualmente o por sugerencia de personas cercanas a ellos. Todos los participantes (tanto los argentinos como los estadounidenses) recibieron un enlace online por medio del cual obtuvieron acceso a las pruebas y al término de consentimiento. Los participantes norteamericanos eran, en su totalidad, estadounidenses, residentes en el mismo país al momento de la realización del estudio. Todos ellos respondieron a las pruebas desde su país de origen.

En ambos grupos, decidimos dejar de lado los datos de aquellos participantes que, además de la lengua inglesa (ya sea como L1 o como L2), hubieran adquirido o estuvieran estudiando alemán, ya que tal idioma comparte patrones similares de VOT a los de la lengua inglesa. Lo mismo ocurrió con los participantes que declararon haber estudiado 0 adquirido otras lenguas cuyo patrón de VOT fuera desconocido o compartido con el inglés y el alemán.

\section{2 Ítems léxicos del experimento}

Los ítems léxicos empleados en las pruebas perceptivas son los mismos utilizados en estudios previos sobre la percepción de los patrones de VOT por brasileños y argentinos (ALVES; MOTTA, 2014; ALVES; ZIMMER, 2015; SCHWARTZHAUPT; ALVES; FONTES, 2015; ALVES; LUCHINI, 2016; 2017a, 2017b; MOTTA-AVILA, 2017). Investigamos la percepción de types del inglés (naturales y manipulados) con estructura CVC, cuya primera consonante era una oclusiva sorda $(/ \mathrm{p} /, / \mathrm{t} /, / \mathrm{k} /)$ y la vocal era anterior y alta, específicamente las vocales (/i/ o /I/), pues, como verificado por Yavas (2008) e Yavas; Wildermuth (2006), estas vocales proporcionan valores más altos de VOT. Utilizamos los types pee ${ }^{4}$, pit, tick, tip, kit, kill. Delimitamos el uso de estas seis palabras ya que abarcan los tres puntos de articulación de las consonantes oclusivas: bilabial (/p/ y /b/), alveolar (/t/ y /d/) y velar (//k/ y /g/). De esta

\footnotetext{
${ }^{4}$ El uso de una palabra con estructura CV (con vocal tensa) se debió al hecho de que, en los estudios previos, también contábamos con una tarea de producción. De esa forma, 'pee' tuvo que ser escogida en detrimento de 'Pete', dado el grafema vocálico al final de la palabra que podría llevar al aprendiz a realizar una vocal epentética de carácter indebido. De hecho, datos de vocales epentéticas se observan en investigaciones previas sobre la influencia del código escrito en la producción en inglés (SILVEIRA, 2016; ZIMMER; SILVEIRA; ALVES, 2009).
} 
forma, hubo dos palabras para cada punto de articulación.

Los estímulos de audio empleados en este estudio perceptivo son los mismos utilizados en los experimentos llevados a cabo en Motta (2014) y Motta-Avila (2017), con estudiantes brasileños de inglés y hablantes norteamericanos. Cada una de estas palabras fue manipulada cuatro veces, generando cinco estímulos diferentes entre ellos: un estímulo natural y cuatro estímulos manipulados, de forma porcentual decreciente, en porciones del $25 \%$ en el $25 \%$ del intervalo total de aspiración (0\%, 25\%, $50 \%, 75 \%, 100 \%$ de la duración total de la aspiración del segmento originalmente aspirado).

\subsection{Grabación y manipulación de los estímulos}

Los estímulos fueron grabados por dos locutoras del sexo femenino. Ambas locutoras son hablantes nativas de inglés estadounidense. La primera locutora proviene de Indianola, estado de lowa, al sur de los Estados Unidos. La segunda locutora es de Western Washington, cerca de la capital del estado (Olympia). Ambas locutoras estudiaron en Brasil y, al momento de hacer las grabaciones, residieron en la ciudad de Porto Alegre.

Los estímulos fueron manipulados manualmente en el software Praat - Versión 5.2.9 (BOERSMA, WEENINK, 2013). La manipulación se dio de la siguiente forma: (i) el estímulo natural fue abierto y salvo en el software Praat como estímulo genuino, considerado como perteneciente al patrón de VOT Positivo, o sea, con el 100\% de la aspiración. Consideramos el $100 \%$ de la aspiración total, realizada de manera natural por las locutoras. Este archivo de audio (en formato .wav), además de ser utilizado en el experimento como estímulo original, dio origen a los estímulos manipulados a partir de él (75\%, 50\%, 25\% y $0 \%$ de aspiración original); (ii) la manipulación de estos estímulos siempre se inició desde el medio de la duración del VOT hacia los bordes, a fin de evitar el corte de otras pistas acústicas no deseadas. A medida que la porción de aspiración disminuía, podía ocurrir que el medio de una de esas "porciones" de VOT quedara con el punto medio cercano de la vocal siguiente. Para evitar manipular la transición formántica de tal vocal, decidimos seleccionar porciones de corte más a la izquierda del punto central de la aspiración, para que tal selección quedara siempre más cercana de la explosión de la consonante en sí que de la vocal que seguía la consonante.

\subsection{Tarea de Identificación}

La tarea de identificación fue la misma empleada en Motta (2014) y Motta-Avila (2017), con oyentes brasileños, nativos del portugués de Brasil, y norteamericanos. El diseño de la tarea de Identificación consistió en 72 tokens por participante, contando 60 estímulos (2 estímulos en cada una de las manipulaciones del punto de articulación, repetidos una vez, totalizando 60 tokens) y 12 catch trials que presentaban el patrón VOT Negativo ${ }^{5}$ (4 para cada punto de articulación).

La tarea consistió en escuchar una palabra de la lengua inglesa (que podría ser manipulada o no) una sola vez e indicar, entre un conjunto de seis posibilidades (/b/, /d/, /g/, /p/ /t/, /k/), con cual consonante se iniciaba dicha palabra. La tarea fue realizada frente a una computadora, individualmente, a través de la plataforma online Survey Gizmo ${ }^{6}$. Las respuestas de los participantes en la tarea de identificación fueron almacenadas por la plataforma para permitir el análisis estadístico posterior. El análisis estadístico se realizó a través del software SPSS - Versión 21. Dado que los resultados de las

\footnotetext{
${ }^{5}$ Como lo explica Silveira (2016), la función de los catch trials es verificar si el participante está realmente prestando atención a la tarea que está realizando. Se espera que los estímulos a los que son expuestos sean identificados con alto grado de aciertos. De no ser así (hecho que no se registró en esta investigación), no deben ser incluidos, porque sus datos estarían sugiriendo poco compromiso con la tarea encomendada. En el caso del presente trabajo, utilizamos el patrón VOT Negativo porque, según lo señalado por estudios anteriores (ALVES, LUCHINI, 2016, 2017a, 2017b), este patrón es característico de las oclusivas sonoras de la variedad de español rioplatense, siendo de fácil identificación por los participantes. Además, la inclusión de estos catch trials garantiza un número mínimo de estímulos en los que las opciones de la tarea referentes a las oclusivas sonoras /b/, /d/, $\mathrm{lg} /$ sean elegidas (dada la posibilidad de que los estudiantes identifiquen todos los demás estímulos manipulados como sordos).

www.surveygizmo.com
} 
pruebas de normalidad de Shapiro-Wilk y Kolmogorov-Smirnov señalaron que no había una distribución normal de los datos, utilizamos tests no paramétricos en el análisis.

\section{Resultados y discusión}

Con base en estudios previos (MOTTA, 2014; SCHWARTZHAUPT; ALVES; FONTES, 2015; ALVES; LUCHINI, 2016, 2017a, 2017b), esperábamos una diferencia entre los patrones de respuesta presentados por oyentes norteamericanos y los estudiantes argentinos. Mientras que los primeros tenderían a identificar los estímulos cada vez menos como sordos a medida que se cortara el VOT, los estudiantes tenderían a presentar mayores índices que los hablantes nativos en la identificación de dichos segmentos como sordos, en función de que el VOT no es la pista prioritaria entre el grupo de extranjeros.

En la siguiente tabla, presentamos los índices de identificación de la consonante como sorda, de acuerdo con cada uno de los patrones de VOT, para cada punto de articulación tomado individualmente. Cabe mencionar que aquí consideramos solamente los índices de identificación de la consonante como sorda en los casos en que los participantes identificaron correctamente el punto de articulación de la consonante (por ejemplo, si una consonante dorsal con aspiración plena fuera identificada como /p/, que también corresponde a un segmento sordo, este dato no sería considerado por tratarse de un error relacionado al punto).
Tabla 01: Índices de identificación de las consonantes como sordas por los participantes argentinos y norteamericanos, y resultado de los tests de MannWhitney entre los dos grupos.

\begin{tabular}{|c|c|c|c|}
\hline $\begin{array}{l}\text { CONSONANTE Y } \\
\text { PROPORCIÓN DE } \\
\text { VOT MANTENIDA }\end{array}$ & Argentinos & $\begin{array}{c}\text { Norte- } \\
\text { Americanos }\end{array}$ & $\begin{array}{l}\text { MANN- } \\
\text { WHITNEY } \\
(U)\end{array}$ \\
\hline$/ p / 100$ & $\begin{array}{l}100 \% \\
94 / 94\end{array}$ & $\begin{array}{c}88,04 \% \\
81 / 92\end{array}$ & $\underset{\star \star \star \star}{47,00}$ \\
\hline$/ p / 75$ & $\begin{array}{c}86,17 \% \\
81 / 94\end{array}$ & $\begin{array}{c}84,78 \% \\
78 / 92\end{array}$ & 227,00 \\
\hline$/ p / 50$ & $\begin{array}{c}88,29 \% \\
83 / 94\end{array}$ & $\begin{array}{c}77,17 \% \\
71 / 92\end{array}$ & 261,50 \\
\hline$/ \mathrm{p} / 25$ & $\begin{array}{c}76,59 \% \\
72 / 94\end{array}$ & $\begin{array}{c}65,21 \% \\
60 / 92\end{array}$ & 243,00 \\
\hline$/ p /$ Oart & $\begin{array}{c}32,97 \% \\
31 / 94\end{array}$ & $\begin{array}{c}18,47 \% \\
17 / 92\end{array}$ & $\underset{*}{174,50}$ \\
\hline$/ t / 100$ & $\begin{array}{l}100 \% \\
94 / 94\end{array}$ & $\begin{array}{c}89,13 \% \\
82 / 92\end{array}$ & 238,00 \\
\hline$/ t / 75$ & $\begin{array}{l}100 \% \\
94 / 94\end{array}$ & $\begin{array}{c}91,30 \% \\
84 / 92\end{array}$ & 238,00 \\
\hline$/ t / 50$ & $\begin{array}{c}86,17 \% \\
81 / 94\end{array}$ & $\begin{array}{c}56,52 \% \\
52 / 92\end{array}$ & $\underset{* \star}{157,50}$ \\
\hline$/ t / 25$ & $\begin{array}{c}65,95 \% \\
62 / 94\end{array}$ & $\begin{array}{c}35,86 \% \\
33 / 92\end{array}$ & $\underset{* *}{134,00}$ \\
\hline /t/ Oart & $\begin{array}{c}40,42 \% \\
38 / 94\end{array}$ & $\begin{array}{c}13,04 \% \\
12 / 92\end{array}$ & $\underset{* *}{154,00}$ \\
\hline$/ \mathrm{k} / 100$ & $\begin{array}{l}100 \% \\
94 / 94\end{array}$ & $\begin{array}{c}85,86 \% \\
79 / 92\end{array}$ & $\underset{*}{214,00}$ \\
\hline /k/ 75 & $\begin{array}{l}100 \% \\
94 / 94\end{array}$ & $\begin{array}{c}85,86 \% \\
79 / 92\end{array}$ & $\begin{array}{c}225,50 \\
* ?\end{array}$ \\
\hline /k/ 50 & $\begin{array}{c}92,55 \% \\
87 / 94\end{array}$ & $\begin{array}{c}78,26 \% \\
72 / 92\end{array}$ & 231,50 \\
\hline$/ k / 25$ & $\begin{array}{c}58,51 \% \\
55 / 94\end{array}$ & $\begin{array}{c}29,34 \% \\
27 / 92\end{array}$ & $\underset{\star \star *}{145,00}$ \\
\hline$/ \mathrm{k} /$ Oart & $\begin{array}{c}10,63 \% \\
10 / 94\end{array}$ & $\begin{array}{c}13,04 \% \\
12 / 92\end{array}$ & 263,00 \\
\hline
\end{tabular}

Nota: ${ }^{*} ? p<.10$ (marginalmente significativo), ${ }^{*} p<.05$, ${ }^{* *} p<.01,{ }^{* * *} p<.001$

Consideremos cada uno de los puntos de articulación individualmente. En lo que se refiere a la oclusiva bilabial, al considerar el grupo de norteamericanos y de argentinos por separado, verificamos que, según el test de Friedman, ambos presentan una diferencia significativa en la identificación de $/ p /$ en función del grado de manipulación de la consonante (norte-americanos: $X^{2}$ $(4)=57,474, p=, 000 ;$ argentinos: $\left.X^{2}(4)=66,20, p=, 00\right)$. Tal diferencia significativa en los datos de los argentinos, en principio, podría ser considerada sorprendente. De hecho, verificamos que, aunque el patrón VOT Cero presenta índices de identificación de /p/ más altos que los verificados entre los nativos (lo 
que será discutido a continuación, en la verificación de los resultados del test de Mann-Whitney, referente a la comparación inter-grupos), debemos considerar que, aunque en menor grado, el VOT ya es tomado como una pista que presenta un cierto grado de relevancia para la atribución del estatus sordo-sonoro. Los resultados de los tests post-hoc de Wilcoxon, con corrección de Bonferroni, señalan diferencias significativas entre las condiciones de manipulación de $100 \%$ vs. $75 \%(Z=-3,000, p=, 003), 100$ vs. $25 \%$ $(Z=-3,456, p=, 001)$, VOT 0 vs. $25 \% \quad(Z=-3,976$, $p=, 000)$, VOT 0 vs. $50 \%(Z=-4,173, p=, 000)$, VOT 0 vs. $75 \%(Z=-4,247, p=, 000)$ y VOT 0 vs. $100 \%(Z=$ -4,338, $p=, 000)$, demostrando que el VOT Cero presenta diferencias significativas con respecto a todos los demás patrones, lo que revela que los estudiantes ya presentan una sensibilidad a este patrón.

Estos hallazgos son, en principio, sorprendentes, porque sugieren un resultado contrario al de los estudios previos realizados con estudiantes argentinos (cf. ALVES; LUCHINI, 2016, 2017a, 2017b). A raíz de estos datos, podemos plantear dos escenarios. El primero se refiere al alto nivel de competencia lingüística de los participantes de este estudio, lo que nos llevaría a concluir que los estudiantes ya están prestando más atención a la pista del VOT. Sin embargo, considerando que los estudiantes investigados en los estudios anteriores también presentaban un nivel de dominio de lengua similar a los investigados en este trabajo, tal posibilidad nos parece menos probable.

El segundo escenario nos parece un tanto más plausible. Es importante considerar que, a diferencia de los estudios previamente mencionados, en la presente tarea de percepción se presentaron a los estudiantes diferentes grados de manipulación de VOT de una oclusiva originalmente sorda. Por su parte, en los estudios anteriores, se presentaron, también, instancias del patrón VOT Cero Natural, con pistas acústicas (tales como fuerza de explosión y F0 de la vocal siguiente) un tanto diferentes de las del patrón manipulado. En otras palabras, en los experimentos anteriores, los estudiantes tenían la posibilidad de asignar mayor valor a otras pistas que no fueran el VOT, dado el Patrón Cero Natural. Sin embargo, en el presente estudio, el único patrón presentado, además de los manipulados, fue el VOT Negativo (catch trials). Si bien tal patrón presenta características acústicas que se diferencian del patrón a partir del cual fueron manipulados estos estímulos (no solo en lo que respecta a la duración de VOT, sino también a la fuerza de explosión y al F0 de la vocal siguiente, entre otros), el número de estímulos con catch trials correspondía solamente a una entre las otras cinco categorías de manipulación investigadas (100\%, 75\%, 50\%, 25\% y VOT 0). De esta forma, no podemos descartar la posibilidad de que los estudiantes pudieron haber prestado mayor atención al VOT de lo que solían hacer, en función del alto índice de estímulos manipulados en la tarea, de modo a ser "forzados" a seleccionar una pista que no necesariamente es su prioridad. A pesar de esta posibilidad, es necesario considerar, sin embargo, que los índices de identificación del patrón VOT 0 como sordo son mucho menores entre los oyentes estadounidenses que entre los estudiantes argentinos.

Con respecto a los resultados del test de Friedman, llevado a cabo con los datos de los estadounidenses, tal resultado ya era esperado. Los tests post-hoc de Wilcoxon, con corrección de Bonferroni, mostraron diferencias significativas entre el patrón del $100 \%$ en comparación con todos los otros patrones [( $100 \%$ vs. $75 \%: Z=-3,435, p=, 001)$, (100\% vs. $50 \%$ : $Z=-3,769, p=, 000)$, (100\% vs. $25 \%$ : $Z=-2,933, p=, 003), \quad(100 \%$ vs. VOT $0: Z=-3,971$, $\mathrm{p}=, 000)$ ], así como entre el patrón VOT 0 en comparación con todos los demás patrones [ $(0 \%$ vs. 25\%: $Z=-3,864, p=, 000)$, (0\% vs. 50\%: $Z=-3,979$, $p=, 000)$, (0\% vs. 75\%: $Z=-3,998, p=, 000)$ ]. Ello nos lleva a sugerir que los oyentes nativos realmente toman el VOT como pista fundamental.

Sin embargo, la observación de los datos de la Tabla 01 apunta a un resultado descriptivo sorprendente, en lo que se refiere al patrón con aspiración plena: mientras que los propios extranjeros ya presentan unos $100 \%$ de identificación de tal 
consonante como /p/, los oyentes nativos tienden a identificarla como /p/ en apenas $88,04 \%$ de los casos (81 de 92 de los estímulos). Al realizar un análisis de las 11 respuestas que no correspondían a la identificación de la opción /p/, verificamos que sólo dos de ellas identificaron la consonante como /b/, habiendo, además, tres identificaciones de la consonante como $/ t /$, dos respuestas de la consonante como /d/ y cuatro como /g/. Conforme previsto en la literatura del inglés (LISKER; ABRAMSON, 1964; KENT; READ, 2015), debido a las acentuadas diferencias de duración del VOT Positivo en las consonantes /p/, /t/ y /k/ (con valores más altos en la dorsal), tal pista acústica puede actuar, también, como secundaria en la identificación de punto de articulación. Eso no ocurre en el español hablado por nuestros participantes, en que /p/ y /t/, por ejemplo, se producen con valores muy cercanos de VOT, ambos mostrando un patrón de VOT Cero (LISKER; ABRAMSON, 1964; ALVES; LUCHINI, $2017 \mathrm{a}, 2017 \mathrm{~b})^{7}$, por lo que tal patrón no es suficiente para una discriminación de punto de articulación. De esta forma, con base en el análisis de las respuestas discrepantes, vemos que, en el caso de los VOTs manipulados (que no necesariamente concuerdan con los valores típicos de su punto de articulación correspondiente), los participantes norteamericanos pueden estar proporcionando sus respuestas tomando como prioritaria una pista que ejerce un rol secundario en la lengua. Esto explica el valor del $100 \%$ entre los estudiantes argentinos y los valores más bajos entre los propios nativos, dado que, en función de la L1 del primer grupo, la duración de VOT no propicia equívocos referentes a la elección de punto de articulación.

En lo que se refiere a la verificación intergrupos, comparamos estadísticamente los índices de identificación de /p/ de los dos grupos para cada una de las condiciones de manipulación de la oclusiva bilabial. Como muestra la Tabla 01, los resultados de los tests de Mann-Whitney señalaron diferencias

\footnotetext{
7 Conforme discutiremos en esta sección, la oclusiva inicial /k/, en el dialecto del español de los participantes de este estudio, presenta una semi-aspiración, con valores de VOT que la diferencian de los encontrados en los demás puntos.
}

significativas únicamente en las condiciones de 100\% (preservación plena) y $0 \%$ (eliminación completa) de VOT. En la condición de $100 \%$ de preservación del VOT de la consonante, tal resultado, como ya fue explicado, nos pareció sorprendente, debido a la toma del VOT como pista para la definición de punto de articulación, por parte de los oyentes norteamericanos. Por su parte, la diferencia encontrada entre los dos grupos en el patrón VOT Cero no se mostró sorprendente, ya que, considerando la poca valoración de la pista acústica entre estudiantes argentinos, esperábamos una diferencia significativa entre los dos grupos. La observación de la Tabla 1 pone de manifiesto que, aunque las diferencias significativas no se han encontrado para las otras tres condiciones de manipulación (25\%, 50\% y 75\%), los índices de identificación de $/ \mathrm{p} /$ son siempre más altos entre los participantes argentinos, confirmando, por lo tanto, el hecho de que el estatus prioritario de VOT necesita ser "aprendido" por los estudiantes a lo largo de su trayectoria de desarrollo del idioma.

Con respecto a la oclusiva alveolar, los tests de Friedman también resaltan diferencias significativas en cada uno de los grupos verificados individualmente (argentinos: $X^{2}(4)=64,839, p=, 000$; norteamericanos: $\left.X^{2}(4)=64,441, p=, 000\right)$. En el caso de los estudiantes argentinos, los tests post-hoc de Wilcoxon, con corrección de Bonferroni, señalan diferencias significativas entre los patrones $100 \%$ vs. $25 \%(Z=-4,027, p=, 000), 100 \%$ vs. VOT $0(Z=-4,049$, $p=, 000), 75 \%$ vs. $25 \%(Z=-4,053, p=, 000), 75 \%$ vs. VOT $0(Z=-3,956, p=, 000), 50 \%$ vs. $25 \%(Z=-3,477$, $p=, 001)$ y $50 \%$ vs. VOT $0(Z=-3,523, p=, 000)$. El hecho de no haberse encontrado diferencias entre los patrones VOT 0 vs. $25 \%, 50 \%$ vs. $75 \%, 50 \%$ vs. $100 \%$ y $75 \%$ vs. $100 \%$ revela una mayor tendencia de identificación de los patrones 50\%, 75\% y 100\% como /t/, en oposición a los patrones con $25 \%$ o VOT 0. Este resultado es diferente al verificado con la bilabial, en el que el VOT Cero contrastaba, incluso, con el patrón de $25 \%$ de aspiración. En el caso de la alveolar, que presenta promedios de VOT más largos que la bilabial en función de su lugar de constricción 
más posterior que la bilabial (cf. LIBERMAN; ABRAMSON, 1964; KENT; READ, 2015), parece que el límite para un cambio entre el tipo de respuestas en la tarea se encuentra en la manipulación del 25\%, y no en el patrón VOT Cero.

En otras palabras, el $25 \%$ de un VOT más largo será tomado como un intervalo corto para una caracterización de la consonante como sorda. Así como en la oclusiva bilabial, en lo que concierne a los resultados del grupo de estudiantes de inglés, los resultados son sorprendentes, ya que demuestran cierta sensibilidad al VOT, posiblemente en función de las posibilidades de explicación ya mencionadas. A pesar de que estos estudiantes no consideran el VOT como pista prioritaria (cf. ALVES; LUCHINI, 2016), los datos revelan que, para estos participantes, esta pista ya cumple un rol importante en la identificación de estos sonidos. Dicha pista debe actuar conjuntamente con otras pistas acústicas referentes a las consonantes oclusivas. A pesar de que esta pista no asume un estado semejante al atribuido por los hablantes nativos, en la presente tarea, juega un rol preponderante para las definiciones de sonoridad, dado que todas las demás pistas son iguales entre todos los patrones manipulados. Sin embargo, cabe señalar que los índices de identificación de /t/ en el patrón de $25 \%$ fueron del 65,95\%, aún mucho (y significativamente) más altos que el $35,86 \%$ de identificación de la consonante como /t/ por los estadounidenses.

Aún con respecto a las comparaciones intragrupo en el grupo de norteamericanos, los tests posthoc de Wilcoxon, con corrección de Bonferroni, señalaron diferencias significativas entre todas las condiciones, con excepción de la comparación entre los patrones $100 \%$ vs. $75 \%{ }^{8}$ [(100\% vs. $50 \%$ : $Z=$

\footnotetext{
8 Aunque la diferencia entre estos dos patrones no es significativa, según los resultados de la estadística descriptiva, la Tabla 01 muestra que el porcentaje de identificación de /t/ referente al patrón $75 \%$ es mayor que el referente al patrón 100 \%. Así como con la oclusiva bilabial, realizamos un análisis de errores que señaló, nuevamente, un bajo índice de identificaciones del estímulo con $100 \%$ de VOT como la contraparte sonora de la oclusiva alveolar (/d/). De hecho, de las 10 respuestas que no se identificaron como /t/, tres fueron identificadas como /d/, tres como /b/, dos como $/ \mathrm{k} /$ y dos como /p/. Confirmamos, nuevamente, que la duración de VOT puede ser tomada como una pista secundaria de punto de articulación
}

$-2,792, p=, 005),(100 \%$ vs. $25 \%: Z=-3,632, p=, 000)$, (100\% vs. VOT 0: $Z=-4,130, p=, 000)$, (75\% vs. $50 \%$ : $Z=-3,448, p=, 001),(75 \%$ vs. $25 \%: Z=-3,858, p=, 000)$, (75\% vs. VOT 0: $Z=-4,263, p=, 000)$, (50\% vs. $25 \%$ : $Z=-2,891, \quad p=, 004), \quad(50 \%$ vs. VOT $0: Z=-3,771$, $p=, 000)$, (25\% vs. VOT 0: $Z=-3,384, p=, 001)]$. Queda clara, por lo tanto, la gran sensibilidad a alteraciones de VOT cuando la consonante manipulada presenta un VOT más largo en su totalidad, como es el caso de la oclusiva alveolar con relación a la bilabial: cuanto mayor el valor pleno del VOT, mayor el intervalo de aspiración extraído y, por consiguiente, mayor el efecto ejercido por la extracción de una porción más larga de la pista acústica. De ello resultará, en el caso de los oyentes que toman tal pista como prioritaria para la distinción de sonoridad, una mayor identificación de la consonante como sonora.

En lo que se refiere a las comparaciones intergrupos, en términos descriptivos, los índices de identificación de /t/ son siempre más altos en las respuestas de los participantes argentinos que en las de los norteamericanos, lo que denota también mayor valoración de tal pista entre los nativos de inglés. En cuanto al análisis inferencial, los tests de MannWhitney revelaron diferencias significativas entre los dos grupos no solo en el patrón VOT Cero (siendo que en este patrón una diferencia significativa también había sido verificada para la labial), $U=$ $154,000, p=, 006$, sino también en las condiciones de preservación de 25\% ( $U=134,000, p=, 002)$ y $50 \%$ $(U=157,500, p=, 007)$ de la duración de VOT de la oclusiva original. En el caso de la alveolar, que presenta un VOT más largo que la bilabial, los oyentes norteamericanos son aún más sensibles a la disminución de la duración de la aspiración, demostrando una sensibilidad significativa mayor que la de los estudiantes argentinos.

Finalmente, con respecto a la oclusiva velar, de modo semejante al verificado en los demás puntos de articulación, los tests de Friedman revelan, en cada uno de los grupos, diferencias significativas entre las condiciones de manipulación (argentinos: $\mathrm{X}^{2}$

entre oyentes norteamericanos (LISKER; ABRAMSON, 1964, KENT; READ, 2015) 
$(4)=84,798, p=, 000$; norteamericanos: $X^{2}(4)=65,283$, $\mathrm{p}=, 000)$. Resaltamos, una vez más, el carácter sorprendente de los resultados de los estudiantes argentinos, dado que, aunque pudieran ser esperadas diferencias en los porcentajes, los resultados de los estudios previos (ALVES; LUCHINI, 2016, 2017a, 2017b) no sugerían diferencias inferenciales significativas entre las condiciones. Nuevamente, destacamos la posibilidad de que, dado que la tarea contaba con diferentes patrones de VOT (y con estímulos que, salvo los catch trials con VOT Negativo, presentaban las mismas características acústicas y se diferenciaban apenas en función del grado de aspiración), tales participantes pudieron haber prestado más atención a esa pista que en contextos no experimentales, en los que deben lidiar con una diversidad de pistas que compiten entre ellas.

Con respecto a las verificaciones post-hoc, los tests de Wilcoxon con corrección de Bonferroni realizados en los datos de los argentinos mostraron diferencias significativas entre las condiciones $100 \%$ vs. $25 \%(Z=-4,078, p=, 000), 100 \%$ vs. VOT $0(Z=$ $-4,516, p=, 000), 75 \%$ vs. $25 \%(Z=-4,078, p=, 000)$, $75 \%$ vs. VOT 0 ( $Z=-4,516, p=, 000), 50 \%$ vs. $25 \%$ ( $Z=$ $-3,803, p=, 000), 50 \%$ vs. VOT $0(Z=-4,370, p=, 000), y$ $25 \%$ vs. VOT 0 ( $Z=-3,878, p=, 000)$. Considerando que las únicas comparaciones que no presentaron diferencias significativas entre las condiciones fueron $100 \%$ vs. $75 \%, 100 \%$ vs. $50 \%$ y $75 \%$ vs. $50 \%$, podemos sugerir que, en el caso de la oclusiva velar, parece haber un punto de corte a partir de la manipulación del $50 \%$ de VOT, de modo que los intervalos de VOT con cortes mayores que la mitad de la duración original ya no tienden a ser identificados como instancias de $/ \mathrm{k} /$. Se trata, por lo tanto, de un comportamiento similar al ya comprobado con la oclusiva alveolar, en oposición al verificado en la oclusiva labial, que presenta duración menor. En otras palabras, los patrones con 50\%, 75\% y $100 \%$ de VOT tienden a ser identificados como / $/$.

Por su parte, en lo que se refiere a los tests de Wilcoxon con corrección de Bonferroni realizados en los datos de los participantes norteamericanos, verificamos diferencias significativas entre las condiciones $100 \%$ vs. $25 \%$ ( $Z=-3,949, p=, 000), 100 \%$ vs. VOT 0 ( $Z=-4,134, p-, 000), 75 \%$ vs. $25 \%$ ( $Z=$ $-4,002, p=, 000), 75 \%$ vs. VOT $0(Z=-4,164, p=, 000)$, $50 \%$ vs. $25 \%(Z=-3,641, p=, 000)$ e $50 \%$ vs. $0 \%(Z=$ $-3,985, p=, 000$ ). Estos resultados son similares a los observados entre los estudiantes argentinos, según los datos previamente presentados, con apenas la diferencia de que, para los norteamericanos, no se observa una diferencia significativa entre los patrones $25 \%$ vs. VOT 0 , dado que los índices de identificación de la consonante como $/ \mathrm{k} /$ en el patrón de $25 \%$ son inferiores al 30\% (cf. Tabla 01). Este comportamiento se condice con la ausencia de una diferencia inferencial significativa entre los dos patrones. Como se discute a continuación, en lo que se refiere a las comparaciones entre grupos, podemos decir que, a medida que la duración del VOT disminuye, pareciera ser que los participantes norteamericanos se muestran más cautelosos para la asignación del estatus sordo.

Los datos de la Tabla 01 también demuestran diferencias significativas entre los dos grupos, de acuerdo con los resultados de los tests de MannWhitney, en los patrones con 100\% ( $U=214,00, p=$ ,033) y 25\% ( $U=145,00, p=, 004)$ de aspiración, habiendo sido encontrados resultados marginalmente significativos en el patrón con 75\% ( $U=225,500, p=$ ,064). En lo que se refiere a la duración total de VOT (100\%), los datos de la Tabla 01 muestran que la diferencia significativa se da en función de una superioridad en los índices porcentuales de identificación de /k/ por argentinos. Nuevamente, resaltamos esta diferencia significativa como un dato sorprendente, dado que esperábamos que ambos grupos presentaran un altísimo grado de identificación en la referida condición. Dada tal constatación, una vez más, observamos los patrones de las 13 respuestas de los participantes que no identificaron tales estímulos como /k/. Esta observación reveló que, de los 13 estímulos no identificados como $/ \mathrm{k} /$, sólo dos fueron reconocidos como la contraparte de la velar sonora $/ g /$. Si se consideran los demás estímulos, uno fue identificado como /p/, tres como 
/b/, tres como /d/ y cuatro como /t/. De nuevo, concluimos que la discrepancia entre el valor de VOT y las demás pistas caracterizadoras de punto (entre otras, la transición formántica de la vocal siguiente) puede llevar a identificaciones equivocadas entre los norteamericanos, dado que la duración de la aspiración también puede contribuir a la identificación del punto de articulación, entre los participantes de dicho grupo.

En lo que se refiere al patrón con 50\%, recordemos que, a pesar de la diferencia significativa observada en este grado de manipulación de la oclusiva alveolar, ninguna diferencia significativa había sido verificada para el punto labial. A diferencia de la consonante con punto de articulación más anterior, en que el corte del $50 \%$ del intervalo de VOT podría ocasionar una caída mayor en los índices de identificación de /t/ por parte de los oyentes norteamericanos $(56,52 \%$, cf. Tabla 01$)$ en el caso de la velar, parece que el corte del $50 \%$ no es capaz de causar una disminución abrupta en la identificación de /k/ por los oyentes nativos (78,26\%, cf. Tabla 01). Este resultado se debe, probablemente, al hecho de que la consonante velar es la que presenta mayor duración de VOT, y que, por consiguiente, incluso el corte de la mitad de la aspiración se muestra suficiente para hacer que los oyentes nativos la consideren como aspirada. Dados estos índices altos de identificación de /k/ por los oyentes nativos, y considerando los ya esperados altos índices de identificación de /k/ por los estudiantes argentinos (que no toman el VOT como pista prioritaria), no se encuentran, por lo tanto, diferencias inferenciales significativas entre los dos grupos.

Por último, diferentemente de lo observado tanto con la oclusiva alveolar como con la bilabial, no se encontraron diferencias significativas entre los dos grupos en el patrón VOT Cero de la oclusiva velar. De hecho, a diferencia de los demás puntos, en que la diferencia entre los dos grupos se daba en función de los índices de identificación de /p/ y /t/ por los estudiantes argentinos, en el caso del punto de articulación en cuestión, los estudiantes presentaron bajos índices porcentuales de identificación de /k/
$(10,63 \%$, conforme Tabla 01$)$. En otras palabras, en el caso de la velar, que, con un punto de articulación más posterior, se caracteriza por presentar mayor intervalo de VOT (cf. LISKER; ABRAMSON, 1964, KENT; READ, 2015), el patrón VOT Cero resulta ser, en la percepción de los argentinos, una pista suficiente para determinar la sonoridad. Este fenómeno se debe a que, de ese punto de articulación, se esperan intervalos de aspiración largos para la consonante sorda, incluso en la L1 de los participantes. Considerando, sobre la base de Alves; Luchini (2017 a, 2017 b), que en el dialecto del español de los participantes de este estudio, la oclusiva /k/ se produce con una semi-aspiración, no es sorprendente que el patrón VOT Cero en el estímulo en cuestión corresponda a una duración menor que la encontrada en la propia L1 de los estudiantes. De hecho, es posible que tal patrón haya sido considerado como un segmento sonoro, por haber sonado "demasiado artificial", ya que una duración tan corta de VOT en /k/ no se encuentra en la L1, ni tampoco en la L2 de los estudiantes. De ello se deriva que la atribución más o menos grande del valor perceptual al VOT dependerá del punto de articulación de la consonante, ya que el VOT presenta diferentes duraciones en la L1 de los participantes.

En suma, los resultados confirman que existen diferencias entre los dos grupos en gran parte de las condiciones probadas, lo que evidencia que los estudiantes argentinos de inglés necesitarán tener en cuenta el VOT para tomar las decisiones adecuadas con respecto a la sonoridad de los segmentos. A pesar de que los hallazgos de esta investigación eran en parte previsibles, pudimos verificar un patrón sorprendente, revelado sobre todo por los análisis intra-grupo con el grupo de argentinos. Si bien el VOT no es la única pista o la pista prioritaria considerada por los estudiantes argentinos, junto con otros indicios, forma parte de las diversas pistas acústicas que estos participantes tienen en consideración al momento de la toma de decisiones. 


\section{Consideraciones finales}

En el presente trabajo investigamos la percepción de oclusivas iniciales del inglés con diferentes patrones de VOT (VOT Positivo con duración total, con $75 \%$ restante de la duración total, $50 \%$ de la duración total, $25 \%$ de la duración total y VOT Cero) por parte de estudiantes argentinos y oyentes norteamericanos. Como resultado de la cooperación entre la UFRGS y la UNMdP, estudios previos (ALVES; LUCHINI, 2016, 2017a, 2017b) revelaban que, a diferencia de los oyentes norteamericanos (SCHWARTZHAUPT; ALVES; FONTES, 2015), los estudiantes argentinos no tomaban el VOT como pista prioritaria (cf. cue weighting, HOLT; LOTTO, 2006; IDEMARU; HOLT; SELTMAN, 2012; LEHET; HOLT, 2017) para las distinciones entre las oclusivas sordas y sonoras. De esta forma, con base en tales trabajos, preveíamos diferencias entre los dos grupos en las identificaciones de cada uno de los patrones testeados (con excepción del patrón 100\%, en el cual esperábamos altos índices de identificación por ambos grupos). En la comparación intra-grupo, dada la sensibilidad al VOT esperada por parte de los oyentes norteamericanos, preveíamos diferencias entre las cinco condiciones. Por otro lado, al especular que los oyentes argentinos enfocarían su atención en otras pistas acústicas, desestimando el VOT, esperábamos altos índices de identificación de la consonante sorda, independiente del grado de manipulación de la consonante, y por consiguiente, no preveíamos diferencias entre las condiciones para este grupo.

Los resultados mostraron diferencias significativas entre los dos grupos en siete de las 15 condiciones probadas, sobre todo en los casos relacionados con el patrón VOT 0 o a cortes más acentuados de la duración del VOT. En el caso de la consonante /k/, cuyo intervalo de VOT tiende a ser más largo, la respuesta al patrón VOT Cero presentó índices bajos de identificación como /k/, sobre todo porque este patrón no se encuentra en la propria L1 de los estudiantes.
Por lo tanto, de lo mencionado anteriormente, concluimos que el análisis del papel más o menos prioritario a ser asumido por la pista de VOT entre estudiantes de inglés depende del punto de articulación de los estímulos. De hecho, en el idioma inglés, cada punto implica una distinción considerable en el intervalo de VOT, lo que tendrá efectos en una mayor o menor detección de tal pista y, por consiguiente, en el valor por ella asumido. En estudios previos (ALVES; MOTTA, 2014, ALVES; ZIMMER, 2015, LUCHINI; ALVES, 2016, LUCHINI; ALVES, 2017a, 2017b), no habíamos considerado análisis individuales por punto de articulación en la discusión de las pruebas perceptivas. El análisis presentado en el presente trabajo, por lo tanto, tiene implicancias para la realización de estudios venideros, ya que sugiere que una mayor sensibilidad al VOT depende de su duración correspondiente al punto de articulación.

En el caso de los estímulos labial y velar que presentaban $100 \%$ de VOT (/p/ y /k/, respectivamente), se han encontrado diferencias significativas no esperadas, sobre todo al considerarse una superioridad en los índices de identificación de tales consonantes por los participantes argentinos. Estos hallazgos confirman los datos provenientes de estudios previos (LISKER; ABRAMSON, 1964; KENT; READ, 2015) en los que se destaca que el VOT, entre hablantes nativos de inglés, puede también actuar, junto con otras pistas, como una pista secundaria para la identificación de punto de articulación. Esto no ocurre entre los oyentes argentinos, cuyas cortas duraciones de VOT, al menos en las oclusivas labial y alveolar, no son suficientes para desarrollar este estatus adicional en dicha pista acústica. En otras palabras, en el inglés norteamericano, el VOT corresponde a la pista primaria para identificación de sonoridad y a la secundaria para la distinción del punto de articulación. Los estudiantes argentinos, específicamente los provenientes de la región en donde se habla el español rioplatense, a su vez, parecen no estar influenciados por la duración de VOT en la asignación de punto; además, parecen no tomar el VOT como 
pista acústica prioritaria para la asignación del estatus de sonoridad de las consonantes iniciales. En cuanto a este último aspecto, como ya lo hemos mencionado (ALVES; LUCHINI, 2016, 2017a, 2017b), es importante que los estudiantes - al menos en este contexto específico - logren tomar mayor conciencia del rol que cumple el VOT, y, por lo tanto, atribuyan a esta pista mayor valoración para poder establecer distinciones de sonoridad.

A pesar de que los participantes argentinos no toman el VOT como pista prioritaria para las distinciones de sonoridad, las comparaciones intragrupos realizadas evidenciaron que tal pista ejerce alguna forma de efecto sobre las decisiones tomadas por los estudiantes. De hecho, aunque, en términos descriptivos, esperábamos algunas diferencias porcentuales entre los índices de identificación de las consonantes sordas en cada una de las condiciones, nos pareció sorprendente el resultado del test de Friedman. Reiteramos, una vez más, la posibilidad de que, en función del bajo número de catch trials (lo que caracteriza una limitación del estudio), la tarea haya "entrenado" implícitamente a los estudiantes a prestar atención al VOT. En otras palabras, por la baja diversidad de pistas acústicas que competían con el VOT en la tarea (una vez que todos los estímulos manipulados presentaban pistas acústicas que, salvo el VOT, eran propias de elementos sordos), los estudiantes pasaron a prestar más atención al VOT de lo que hubieran hecho en condiciones no experimentales, o en condiciones experimentales con mayor variabilidad de pistas. Dicha variabilidad de pistas fue propiciada en los estudios de Alves; Luchini (2016, 2017 a, 2017b), en los que, además de los patrones VOT Cero Manipulado, VOT Positivo y VOT Negativo, contábamos, también, con un VOT Cero no manipulado (que, a pesar de mostrar la misma duración de VOT del patrón manipulado, presentaba las demás pistas, tales como F0 y fuerza del burst, distintas del patrón equivalente en términos de VOT).

Independientemente de la justificación para las diferencias significativas encontradas, los resultados demuestran que, aunque no lo tomen de forma prioritaria como lo hacen los oyentes nativos, los estudiantes argentinos tienen el VOT en consideración en sus identificaciones, de forma integrada a otras pistas acústicas. En este sentido, resaltamos que, aunque cada lengua tome una pista como prioritaria, el proceso perceptual involucra la acción integrada de una serie de pistas, prioritarias o secundarias, que, al actuar juntas, dan cuenta de la complejidad de dicho proceso (incluso, a compensarse en casos de ruido o de información ambigua, como en el caso de las identificaciones erróneas referentes al patrón 100\% por parte de los estadounidenses, en el que el VOT resultó asumir un papel especial en las distinciones de punto).

Los resultados del presente estudio, por lo tanto, abren nuevos caminos en nuestra agenda de cooperación, y resaltan la necesidad de realizar nuevos estudios en los que se investigue el papel del punto de articulación en las decisiones perceptivas sobre sonoridad, así como el rol de las demás pistas acústicas que están actuando en conjunto con el VOT, en la identificación de sonoridad por los argentinos. A partir de esos resultados, reafirmamos nuestra convicción de que serán muchos los estudios provenientes de la cooperación internacional entre nuestros grupos de investigación, realizados tanto en el contexto brasileño como en el argentino.

\section{Referências}

ABRAMSON, Arthur S.; WHALEN, Douglas H. Voice Onset Time (VOT) at 50: theoretical and practical issues in measuring voicing distinctions. Journal of Phonetics, v. 63, p. 75-86, 2017.

ALVES, Ubiratã Kickhöfel; KAMPFF, Felipe Rodrigues. Efeitos de longo prazo do treinamento perceptual na percepção e produção das plosivas iniciais surdas do inglês por estudantes brasileiros: implicações para o ensino de pronúncia. Ilha do Desterro, v. 72, n. 3, (no prelo).

; LUCHINI, Pedro Luis. Percepción de la distinción entre oclusivas sordas y sonoras iniciales del inglés (LE) por estudiantes argentinos: datos de identificación y discriminación. Revista Lingüística (ALFAL), v. 32, p. 25-39, 2016.

Effects of perceptual training on the identification and production of word-initial voiceless stops by Argentinean learners of English. Ilha do Desterro, v. 70, n. 3, p. 15-32, 2017 a. 
Percepción y producción de segmentos oclusivos sordos y sonoros del inglés (LE) por argentinos. In: BORTOLON, Mariela; PÉREZ, Elena del C.; MONTES, Mariana; FICARRA, Paula García. (eds.). Aportes a la Lingüística Cognitiva. Córdoba, Argentina: Editorial de la UNC, p. 9-26, 2017b.

; MOTTA, Camila Saviczki. Focusing on the right cue: Perception of voiceless and voiced stops in English by Brazilian learners. Phrasis - Studies in Language and Literature, v. 50, p. 31-50, 2014.

; SCHERESCHEWSKY, Laura Castilhos; LUCHINI, Pedro Luis. L2 development and L1 attrition in an L1dominant environment: analyzing Voice Onset Time in L1 Spanish and L2 English. Estudos da Língua(gem), v. 17, n.1 (no prelo).

; ZIMMER, Márcia Cristina. Perception and production of English VOT patterns by Brazilian learners: the role of multiple acoustic cues in a DST perspective. Alfa Revista de Linguística, v. 59, p. 157180, 2015.

BOERSMA, Paul; WEENINK, David. Praat: Doing Phonetics by Computer - Version 5.2.9. 2013.

CELCE-MURCIA, Marianne et al. Teaching Pronunciation: a course book and reference guide. Cambridge: Cambridge University Press, 2010, 556 p.

FRANÇA, Karoline Veiga. A Aquisição da Aspiração. Curitiba: Appris, 2015, 115 p.

HOLT, Lori L.; LOTTO, Andrew J. Cue weighting in auditory categorization: implications for first and second language acquisition. Journal of the Acoustical Society of America, v. 119, n. 5, p. 3059-3071, 2006.

IDEMARU, Kaori; HOLT, Lori L.; SELTMAN, Howard. Individual differences in cue weights are stable across time: the case of Japanese stop lengths. Journal of the Acoustical Society of America, v. 132, n. 6, p. 3950-3964, 2012.

JENKINS, Jennifer. The Phonology of English as an International Language. Oxford: Oxford University Press, 2000, $258 \mathrm{p}$.

KENT, Ray; READ, Charles. Análise Acústica da Fala. Tradução de Alexsandro Rodrigues Meireles. $1^{\underline{a}}$ ed. São Paulo: Cortez, 2015, 504 p.

LEHET, Matthew; HOLT, Lori L. Dimension-based statistical learning affects both speech perception and production. Cognitive Science, v. 41, n. S4, p. 885912, 2017.

LUCHINI, Pedro Luis; GARCÍA JURADO, María Amalia; ALVES, Ubiratã Kickhöfel. Fonética y Fonología Articulación entre enseñanza e investigación. Mar del Platal: Editorial de la Universidad Nacional de Mar del Plata, 2015.
LISKER, Leigh; ABRAMSON, Arthur. A cross-language study of voicing in initial stops: acoustical measurements. Word, v. 20, p. 384-422, 1964.

MOTTA, Camila Saviczki. Percepção de plosivas surdas do inglês sob múltiplas manipulações de Voice Onset Time (VOT) em tarefa de identificação por brasileiros e americanos. Trabalho de Conclusão de Curso (Graduação em Letras). 91 f. Porto Alegre: Universidade Federal do Rio Grande do Sul, 2014.

MOTTA-AVILA, Camila. A formalização fonéticofonológica da percepção de plosivas surdas sob múltiplas manipulações de Voice Onset Time (VOT) por brasileiros e americanos à luz do modelo 'BIPHON'. Dissertação (Mestrado em Letras). $284 \mathrm{f}$. Pelotas: Universidade Católica de Pelotas, 2017.

PRESTES, Suzana. Produção de consoantes oclusivas iniciais do inglês por falantes nativos do $P B$. Dissertação (Mestrado em Letras). 139 f. Curitiba: Universidade Federal do Paraná, 2013.

REIS, Mara; NOBRE-OLIVEIRA, Denize. Effects of perceptual training on the identification and production of English voiceless plosives aspiration by Brazilian EFL learners. In: RAUBER, Andreia Schurt; WATKINS, Michael; BAPTISTA, Barbara Oughton (eds.). New Sounds 2007: Proceedings of the Fifth International Symposium on the Acquisition of Second Language Speech. Florianópolis: Universidade Federal de Santa Catarina, 2008, p. 372-381.

SCHWARTZHAUPT, Bruno Moraes. Factors influencing Voice Onset Time: analyzing Brazilian Portuguese, English and Interlanguage data. Trabalho de Conclusão de Curso (Graduação em Letras). 65 f. Porto Alegre: Universidade Federal do Rio Grande do Sul, 2013.

Testing intelligibility in English: the effects of Positive VOT and contextual information in a sentence-transcription task. Dissertação (Mestrado em Letras). 86 f. Porto Alegre: Universidade Federal do Rio Grande do Sul, 2015.

ALVES, Ubiratã Kickhöfel; FONTES, Ana B. Arêas da Luz. The role of L1 knowledge on L2 speech perception: investigating how native speakers and Brazilian learners categorize different VOT patterns in English. Revista de Estudos da Linguagem, Belo Horizonte, v. 23, n.2, p. 311-334, 2015.

SILVEIRA, Rosane. The influence of pronunciation instruction on the perception and production of English word-final consonants. Florianópolis: DLLE, CCE, UFSC, 2016, 277 p.

YAVAS, Mehmet. Factors influencing the VOT of English long lag stops and interlanguage phonology. In: RAUBER, Andreia Schurt; WATKINS, Michael; BAPTISTA, Barbara Oughton (eds.). New Sounds 2007: Proceedings of the Fifth International Symposium on the Acquisition of Second Language Speech. Florianópolis: Universidade Federal de Santa Catarina, 2008, p. 492-498. 
; WILDERMUTH, Renée. The effects of place of articulation and vowel height in the acquisition of English aspirated stops by Spanish speakers. International Review of Applied Linguistics, v. 44, p. 251-263,2006.

ZIMMER, Márcia Cristina. A transferência do conhecimento fonético-fonológico do Português Brasileiro (L1) para o Inglês (L2) na recodificação leitora: uma abordagem conexionista. Tese (Doutorado em Letras). 194 f. Porto Alegre: Pontifícia Universidade Católica do Rio Grande do Sul, 2004.
; SILVEIRA, Rosane; ALVES, Ubiratã Kickhöfel. Pronunciation Instruction for Brazilians: Bringing Theory and Practice Together. 1. ed. Newcastle upon Tyne: Cambridge Scholars Publishing, 2009, 220 p.

\section{COMO CITAR ESSE ARTIGO}

ALVES, Ubiratã Kickhöfel; LUCHINI, Pedro Luis; MOTTA-AVILA, Camila. ¿'Pee' o 'Bee’? Identificación por oyentes argentinos y norteamericanos de oclusivas iniciales del inglés con diferentes duraciones manipuladas de Voice Onset Time. Signo, Santa Cruz do Sul, v. 44, n. 81, dez. 2019. ISSN 1982-2014. Disponível em: $<$ https://online.unisc.br/seer/index.php/signo/article/view/13967>. Acesso em: doi: https://doi.org/10.17058/signo.v44i81.13967. 